\title{
Respiratory rehabilitation in interstitial lung diseases
}

\begin{abstract}
Introduction: The interstitial lung diseases (ILD) are mainly characterized by: dry cough, dyspnea and exercise desaturation. These symptoms are due, among other causes, to the lack of pulmonary distensibility and to the alteration of the gas exchange. The objective of this study is to demonstrate the effects that a respiratory rehabilitation program (RRP) has in a group of patients with ILD.

Method: prospective cohort study that was approved by the Maule Health Service Ethics Committee and all Patients signed an informed consent, which were 10 (3 men, 7 women), age: $72.4 \pm 7.9$ years with diagnosis of ILD, specifically 7 with usual interstitial pneumonia(UIP), of which three are associated with rheumatoid arthritis(RA), 3 to idiopathic pulmonary fibrosis(IPF) and 1 to chronic hypersensitivity pneumonitis(CHP), 2 with nonspecific interstitial pneumonia (NSIP) and 1 cryptogenic organizing pneumonia(COP), which were subjected to a RRP based on aerobic training of the respiratory and peripheral musculature, during 36 sessions of 60 minutes each, 3 times a week. The measured variables pre and post training were: body mass index(BMI), forced vital capacity(FVC), distance walked through the six minute walking test(6MWT), dyspnea through the modified scale of the medical research council(MMRC), strength of the inspiratory musculature through the maximal inspiratory pressure(MIP), initial saturation of oxygen(ISO2), final saturation of oxygen(FSO2), desaturation of oxygen(DO2) measured with pulse oximetry, diffusing capacity of the lung for carbon monoxide(DLCO) and quality of life through Saint George Respiratory Questionnaire (SGRQ). The statistical analysis was Carried Out with the program SPSS version 23.
\end{abstract}

Results: significant changes $(\mathrm{p}<0.05)$ in: FVC, 6MWT, mMRC, MIP, and SGRQ Non-significant changes ( $\mathrm{p}>0.05)$ in: BMI, ISO2, FSO2, DO2, and DLCO.

Conclusion: our RRP improved lung function, exercise capacity, dyspnea, respiratory muscle strength and quality of life of the subjects intervened.

Keywords: rehabilitation, interstitial lung disease, quality of life
Volume 2 Issue 5 - 2017

Jiménez J, Rojas C, Lobos M,Vásquez J

CESFAM La Florida Talca, Chile

Correspondence: Klgo Jaime Jimenez S, CESFAM La Florida Talca, Chile, Email jaime_jimenez25@hotmail.com

Received: November 27, 2017 | Published: December 27, 2017

\section{Introduction}

ILDs are a heterogeneous group of parenchymal lung disorders, which are classified together because they present similar clinical, radiological, physiopathological and anatomopathological characteristics. ${ }^{1}$ The etiology of the ILD is varied, with more than 150 known causes, but only about in $35 \%$ of the cases a causative agent can be identified. ${ }^{2}$ Clinically ILDs are generally characterized by dry cough, dyspnea, fine crackles at auscultation and oxygen desaturation during exercise. ${ }^{3}$ As a consequence, patients suffering from this disease tend to be sedentary with functional limitation and deterioration of quality of life. Formerly, limiting the exercise in these patients was recommended. ${ }^{4}$ However, currently available evidence suggests that respiratory rehabilitation (RR) can be an effective and safe treatment. ${ }^{5}$ Sometimes, these diseases are rarely talked about considering that they have a lower prevalence compared to asthma and COPD. Of all the ILD, idiopathic pulmonary fibrosis (IPF) is the most common, its incidence is not certainly known, but several studies have suggested figures between 6.8 and 16.3 per 100,000 inhabitants. The prevalence is also not known with certainty and truthfully, there is not reliable data in our country, but a recent US study proposes a figure between 14 and 42.7 per 100,000 inhabitants depending on whether strict or broader diagnostic criteria are used. ${ }^{6}$ The IPF is progressive and unfortunately does not have an effective drug therapy and has a worse average survival rate than many cancers, which is estimated to be 3 to 5 years after diagnosis. ${ }^{7}$ The quality of life of these patients is chaotic, slowly reducing their functionality; many end using home oxygen and eventually die of respiratory failure. ${ }^{8}$ Due to the poor prognosis for this disease patients often end up terminally ill. ${ }^{9}$ Nevertheless, it is known that ILDs other than IPF or UIP pattern generally have a good prognosis, and if early diagnosis and treatment are obtained, their condition could improve..$^{10,11}$ Thus, we think that it is extremely necessary to implement non-pharmacological measures in these patients, such as supervised aerobic physical activity through a RRP. ${ }^{12,13}$ The evidence - based medicine has shown positive results in terms of quality of life, dyspnea, and exercise capacity of patients who have undergone a RRP. ${ }^{14-17}$ The objective of this study is to demonstrate the effects of a respiratory rehabilitation program designed in primary care based on education, training of peripheral and respiratory musculature, respiratory physiotherapy and functional activities in a group of patients with ILD. Our research question is: Are there significant changes in the 6MWT, FVC, DLCO, initial oxygen saturation, final oxygen saturation, oxygen desaturation, dyspnea, MIP and quality of life of the patients with ILD after completing a respiratory rehabilitation program in primary care?

\section{Methodology}

The study protocol was approved by the scientific ethics committee of the Maule health service and all subjects involved signed the 
informed consent. Prospective cohort study of 10 patients ( 3 men, 7 women) with a mean age \pm SD of $72.4 \pm 7.5$ years. Inclusion criteria were: patients with ILD diagnosed through HRCT of the thorax or histologically with lung biopsy, oxygen saturation at rest with environmental air greater than $90 \%$, without medical contraindications to perform physical activity, compensated comorbidity, medical prescription and most importantly, that they had a desire to participate in the program.

Exclusion criteria were; decompensated heart failure, any other coexistent chronic respiratory disease such as bronchial asthma and any other significant comorbidity that would limit patients to participate in program activities. The variables measured pre and post training were: body mass index(BMI), forced vital capacity(FVC), six minutes walking test $(6 \mathrm{MWT})^{18}$ dyspnea through the modified scale of medical research council(MMRC), maximal inspiratory pressure(MIP), initial saturation of oxygen(ISO2), final saturation of oxygen(FSO2), and desaturation of oxygen(DO2), diffusing capacity of the lung for carbon monoxide(DLCO) ${ }^{19}$ and quality of life through the Saint George 's Respiratory Questionnaire(SGRQ) ${ }^{20}$ with its four variables: symptoms(SGRQ-S), activity(SGRQ-A), impact(SGRQ-I) and total score(SGRQ-T). ${ }^{21}$ The statistical analysis was performed using the program SPSS, version 23, using the student $t$ test for related samples, using $\mathrm{P}<0.05$ for significant changes. The duration of the program was 12 weeks ( 3 months) with one hour sessions three times a week.

\section{Type session}

Each session, vital signs were initially evaluated (BP, HR, RR, saturation of oxygen and dyspnea through the Borg scale), then oxygen was connected to those patients who had it indicated to exercise (which were three), followed by warm-up exercises, then exercises for upper extremities and lower extremities, active-resisted with red there band. ${ }^{22}$ Patients worked the muscles: Deltoids, Pectorals, Brachial Biceps, Crural Quadriceps, Gluteus medius and Triceps Sural. (For each muscle they worked three sets of 10 repetitions for the first month, three sets of 12 repetitions for the second month and three sets of 14 repetitions for the third month).

Then we performed kinetic techniques of pulmonary re-expansion with a three seconds apnea in maximum inspiration combined with diaphragmatic re-education (10 repetitions). Then the inspiratory musculature was worked with the threshold valves at $30 \%$ of the MIP obtained at CRF for the first month, then it was increased to $40 \%$ of the MIP for the second month and finally to $50 \%$ for the third month (three sets of 10 repetitions each). In addition patients were indicated to use the valve at home, for $10 \mathrm{~min} /$ day for the first month, $15 \mathrm{~min} /$ day for the second month and $20 \mathrm{~min} /$ day for the third month. Then the program continued with $10 \mathrm{~min}$ of education where patients had the opportunity to ask questions and finally it ended with functional activities. $^{23}$

\section{Functional activities}

During the first four weeks, the program ended with a 5 minutes free walk through a 10 meters corridor. During the second month patients had to walk a set distance with a bag of 2 kilograms of weight $(20 \mathrm{~m}$ for 5 th week, $40 \mathrm{~m}$ the 6 th week, $60 \mathrm{~m}$ the $7^{\text {th }}$ week and $80 \mathrm{~m}$ the 8 th week). And during the third month, a distance of $100 \mathrm{~m}$ remained with a bag of 3 kilograms of weight. Then vital signs were re-evaluated, mainly saturation of oxygen, HR and dyspnea through the Borg scale.
The program ended with cooldown exercises (elongations, breathing exercises), each session lasted approximately 60 minutes. ${ }^{24}$

\section{Results}

Significant changes $(p<0.05)$ were obtained in: FVC, 6MWT, dyspnea, MIP and quality of life with its four variables: symptoms (SGRQ-S), activity (SGRQ-A), impact (SGRQ-I) and total score (SGRQ-T). No significant changes ( $p>0.05$ ) were obtained in: BMI, DLCO, ISO2, FSO2 and DO2 (Table 1). Groups showed no significant differences from one another: patients with non-UIP ILD did not have better or worse results with respect to subjects with UIP, and UIP patients associated with RA did not have better or worse results with respect to the UIP patients without connective tissue diseases.

Table I Variable results evaluated before and after training

\begin{tabular}{|c|c|c|c|}
\hline Variables & Pre $\operatorname{rrp} \mathbf{N}=10$ & Post $\operatorname{rrp} \mathbf{N}=10$ & Value $\mathbf{P}$ \\
\hline $\mathrm{BMI}\left(\mathrm{kg} / \mathrm{m}^{2}\right)$ & $28.17 \pm 5.40$ & $26.64 \pm 4.7$ &, 195 \\
\hline FVC(L) & $2.03 \pm 0.58$ & $2.45 \pm 0.71$ &, 001 \\
\hline FVC(\%) & $79.70 \pm 27.46$ & $96.80 \pm 34.74$ &, 002 \\
\hline 6MWT $(m)$ & $336.92 \pm 94.67$ & $367.72 \pm 77.17$ & 027 \\
\hline Dyspnea m MRC(0-4) & $2 \pm 0$ & $\mathrm{I} \pm 0.47$ &, 000 \\
\hline $\operatorname{MIP}\left(\mathrm{cm} \mathrm{H}_{2} \mathrm{O}\right)$ & $62.50 \pm 17.81$ & $76.30 \pm 22.44$ & ,004 \\
\hline SGRQ-S(\%) & $46.44 \pm 16.12$ & $20.20 \pm 10.11$ &, 000 \\
\hline SGRQ-A(\%) & $75.90 \pm 14.40$ & $43.06 \pm 14.43$ &, 000 \\
\hline SGRQ-I(\%) & $32.48 \pm 8.30$ & $17.40 \pm 7.60$ &, 000 \\
\hline SGRQ-T(\%) & $47.96 \pm 9.49$ & $25.31 \pm 8.05$ &, 000 \\
\hline $\mathrm{DLCO}(\mathrm{ml} / \mathrm{min} / \mathrm{mm} \mathrm{Hg})$ & $1 \mathrm{I} .0 \mathrm{I} \pm 3.54$ & $10.99 \pm 2.77$ & ,967 \\
\hline DLCO(\%) & $54.70 \pm 16.61$ & $54.60 \pm|3.5|$ & 973 \\
\hline ISO2(\%) & $96.60 \pm 1.07$ & $96.80 \pm 1.39$ & 443 \\
\hline FSO2(\%) & $89.70 \pm 5.25$ & $87.10 \pm 6.15$ & , 153 \\
\hline DO2(\%) & $6.90 \pm 4.62$ & $9.70 \pm 5.33$ & 147 \\
\hline
\end{tabular}

\section{Comments}

There are numerous studies that support that patients with nonUIP ILD respond better to respiratory rehabilitation programs than UIP patients, and there is evidence that patients with UIP associated with RA have better results with physical activity versus pure UIP patients. ${ }^{25}$ However, this trend did not occur in our study and we believe that this may have been due to the small size of our sample. On the other hand, although we ambitiously wanted to improve the diffusion of oxygen or at least reduce the desaturation of oxygen with exercise, we found the opposite once the program ended. That is, decreased DLCO and therefore oxygen supply ${ }^{26}$ and also patients desaturated more, showing lower final oxygen saturation and a greater desaturation of it post intervention. Then: How do we explain that patients increased the meters walked in the walk test? Although we are aware that the increase of the meters walked may have been due to multivariate variables such as increased patient motivation and training of the respiratory muscles and peripheral post intervention ${ }^{27,28}$ we dare to say that this result may be due to improved oxygen 
performance, i.e. peripheral oxygen extraction increased in the muscle, when measuring the oxygen saturation with a pulse oximetry we are measuring the concentration of oxygen bound to hemoglobin, but unfortunately we do not know the oxygen concentration that would be attached to the myoglobin in a muscle level. Keyser et. al. measured this in Virginia, USA, in 2015, through near infrared spectroscopy in the Gastrocnemius muscles of the right leg of a group of patients with ILD and demonstrated that aerobic exercise increases the oxygen binding to myoglobin, concluding that physical exercise improves oxygen performance since using the same or even less oxygen contribution than in the pre training, subjects increased exercise capacity. ${ }^{29}$ Although we do not have the same sophisticated measurement, we have no other answer to what happened than to think that our RRP improved the oxygen performance and its absorption at the peripheral level as it occurred in the study of Keyser. We cannot otherwise explain the significant increase in exercise capacity after the intervention of our subjects, since they used a lower oxygen supply than they did to start training. However we recognize the limitations of the study, such as the fact of not being able to measure the oxygen binding to myoglobin, thus we pose the question for the justification for the increase in exercise capacity observed in our study.

\section{Conclusion}

Our RRP improved lung function, exercise capacity, dyspnea, respiratory muscle strength and quality of life of the subjects. Nonetheless, oxygen diffusion did not improve, echoing other studies conducted in this area. ${ }^{30}$ But we firmly believe that the respiratory rehabilitation (besides all the aforementioned benefits), even if it does not improve gas exchange, it at least improves oxygen performance during exercise.

\section{Acknowledgements}

None.

\section{Conflict of interest}

I do not have acknowledgment and I do not have conflict of interest.

\section{References}

1. Kadoch M, Cham M, Beasley M, et al. Idiopathic Interstitial Pneumonias: A radiology-pathology correlation based on the revised 2013 american thoracic society-european respiratory society classification system. Curr Probl Diagn Radiol. 2015;44(1):15-25.

2. Xaubet A, Ancochea J, Blanquer R, et al. Diagnosis and treatment of diffuse interstitial lung diseases. Arch Bronconeumol. 2003;39(12):580600 .

3. Xaubet A, Molina M, Ancochea J. New classification of idiopathic interstitial pneumonias. Respiratory Medicine. 2014;7(2):21-28.

4. Güell M, Díaz S, Rodríguez G, et al. SEPAR regulations. Respiratory rehabilitation. Arch Bronconeumol. 2014;50(8):332-344.

5. Molina J, Trigueros J, Quintano J, et al. Idiopathic pulmonary fibrosis: a challenge for primary care. Semergen. 2014;40(3):134-142.

6. Undurraga A. Idiopathic pulmonary fibrosis. Rev Med Clin Condes. 2015;26(3):292-301

7. Swigris J, Fairclough D, Morrison M, et al. Benefits of Pulmonary Rehabilitation in Idiopathic Pulmonary Fibrosis. Respir Care 2011;56(6):783-789.
8. Pinheiro S, Barbosa R, Guedes B, et al. Desaturation instance ratio: A new concept for a functional assessment of interstitial lung diseases. Clinics. 2010;65(9):841-846.

9. Strookappe B, Elfferich M, Swigris J, et al. B enefits of physical training in Patients With idiopathic or end - stage sarcoidosis - related pulmonary fibrosis: a pilot study. Sarcoidosis Vasc Diffuse Lung Dis. 2015;32:43-52.

10. Holland A, Hill C, Glaspole I, et al. Predictors of interstitial pulmonary rehabilitation following benefit for lung diseases. Respiratory Medicine. 2012;106(3):429-435.

11. Dowman L, Donald Ch, Hill C, et al. The benefits of exercise training in interstitial lung disease: protocol for a multicenter randomized controlled trial. BMC Pulmonary Medicine. 2013;13:1-8.

12. Betancourt J, Hurtado H. Effects of a pulmonary rehabilitation program in patients with diffuse interstitial disease. Physiotherapy. 2015;356:1-7.

13. Spielmanns M, Gloeckl R, Schmoor C, et al. Effects on pulmonary rehabilitation in patients with COPD or ILD: A retrospective analysis of clinical and functional predictors with particular emphasis on gender. Respiratory Medicine. 2016;113:8-14.

14. Dowman L, McDonald Ch, Hill C, et al. The evidence of benefits of exercise training in interstitial lung disease: a randomized controlled trial. Thorax. 2017;72(7):610-619.

15. Rifaat N, Anwar E, Ali M, et al. Value of pulmonary rehabilitation In Patients With idiopathic pulmonary fibrosis. Egyptian Journal of Chest Diseases and Tuberculosis. 2014;63(4):1013-1017.

16. Gupta K, Kumar R, Gaur S. Effect of a pulmonary rehabilitation domiciliary progamme on disability in patients with interstitial lung diseases. Indian J Chest Dis Allied Sci. 2007;49:213-217.

17. Nishimaya $\mathrm{O}$, Kondoh $\mathrm{Y}$, Kimura $\mathrm{T}$, et al. Effects of pulmonary rehabilitation in patients with idiopathic pulmonary fibrosis. Respirology. 2008;13(3):394-399.

18. Gochicoa L, U Mora, Guerrero S, et al. Walk test 6 minutes: recommendations and procedures. Cir Neumol Thorax. 2105;74(2):127136.

19. Caviedes I, Borzone G, Briceño C, et al. Standardization test diffusibility of carbon monoxide. Rev Chil Enf Respir. 2014;30:145-155.

20. Aguilar M, M Sotelo, Lara A, et al. Reproducibility st. george's respiratory questionnaire in the spanish version of mexican patients with chronic obstructive pulmonary disease. Rev Inst Nal Enf Resp Mex. 2000;13(2):85-95.

21. Tabaj G Quadrelli S, Grodnipzky L, et al. Quality of life in patients with idiopathic pulmonary fibrosis. Am Rev Resp Med. 2012;3:108-116.

22. Salhi B, Troosters T, Behaegel M, et al. Effects of patients with pulmonary rehabilitation in restrictive lung diseases. Chest. 2010;137(2):273-279.

23. Betancourt J, Torres N, Hurtado H. pulmonary rehabilitation in patients with diffuse interstitial lung disease: Case study. Rev Mov Sci. 2015;9(2):6-14.

24. Olson AL, Swigris J, Belkin A, et al. Physical functional capacity in idiopathic pulmonary fibrosis: performance characteristics of the continuous-scale physical function performance test. Expert Rev Respir Med. 2015;9(3):361-367.

25. Solomon J, Ryu J, Tazelaar H, et al. Fibrosing interstitial pneumonia predicts survival in patients with rheumatoid arthritis - associated interstitial lung disease (RA-ILD). Respiratory Medicine. 2013;107(8):1247-1252.

26. Gutierrez M, Sanchez M, Cuellar M, et al. compromised pulmonary function in patients with idiopathic pulmonary fibrosis. Rev Chil Enf Respir. 2008;24:279-285. 
27. Vainshelboim B, Oliveira J, Yehoshua L, et al. Exercise training - based pulmonary rehabilitation program is clinically beneficial for idiopathic pulmonary fibrosis. Respiration. 2014;88(5):378-388.

28. Rammaert B, Leroy S, Cavestri B, et al. Home - based in idiopathic pulmonary rehabilitation pulmonary fibrosis. Revue des Maladies Respiratoires. 2011;28(7):52-57.
29. Keyser RE, Woolstenhulme JG, Chin LM, et al. Cardio respiratory function before and after aerobic exercise training in patients with interstitial lung disease. Journal of Cardiopulmonary Rehabilitation and Prevention. 2015;35(1):47-55.

30. Dell S, Castilian M, Dannaoui $M$, et al. Long-term respiratory rehabilitation in patients with diffuse interstitial lung disease on the lung transplant list. Case series. RAMR. 2006;3:279-283. 